\title{
Finite-size corrections to Fermi's golden rule
}

\author{
Kenzo Ishikawa*广 \\ Hokkaido University \\ E-mail: ishikawadparticle.sci.hokudai.ac.jp \\ Yutaka Tobita \\ Hokkaido University \\ E-mail: tobitadparticle.sci.hokudai.ac.ip
}

The transition process in quantum mechanics has been studied with the probability $\Gamma T$ proportional to the time interval $\mathrm{T}$ between the initial and final states, where $\Gamma$ is the rate. Recently it was found that the constant term $P^{(d)}$ is added as $P=\Gamma T+P^{(d)} . P^{(d)}$ has been ignored for a macroscopic T, but that is not ignoable in various systems. We find $P^{(d)}$ in such processes that $\Gamma \approx 0$ and show that it has wave-like properties and leads unusual quantum phenomena. $P^{(d)}$ is large in magnitude and in spatial size for light particles and has universal properties in relativisticaly invariant systems. Implications to processes of neutrinos in beta decays and gamma rays in various radiative transitions are presented.

XV International Conference on Hadron Spectroscopy-Hadron 2013

4-8 November 2013

Nara, Japan

* Speaker.

${ }^{\dagger}$ A footnote may follow. 


\section{Boundary conditions of transition processes}

A wave length of a particle of a momentum $p$ given by Planck constant $h$ as $\frac{\hbar}{p}$, where $\hbar=$ $\frac{h}{2 \pi}$, is of microscopic size, and wave phenomena have been considered to arise inside this length. Scatterings outside this region have been treated with an independent scattering hypothesis, namely successive reactions occur independently. Our recent study [W] shows that in various cases wave properties appear in regions $L \leq L_{0}, L_{0}=\frac{\hbar}{m c} \times \frac{E}{m c^{2}}$, where $m$ and $E$ is a particle's mass and energy, and the independent hypothesis is not valid. $L_{0}$ is much longer than $\frac{\hbar}{p}$ for small mass and high energy. This phenomenon is caused by many body interactions without obstacles and its pattern is determined by the fundamental constants.

Scattering amplitude in $L \gg L_{0}$ is rigorously formulated with wave packets, and approximations with plane waves combined with a damping factor $e^{-\varepsilon|t|}$ of a positive and infinitesimal $\varepsilon$ in an interaction Hamiltonian work normaly. In $L \leq L_{0}$, probabilities of the events that the final states are detected at $L=c T$ is given in the form,

$$
P=\Gamma T+P^{(d)},
$$

where $\Gamma$ is computed with Fermi's golden rule and insensitive to boundary conditions. Now, $P^{(d)}$ is computed differently and sensitive to boundary conditions at finite $T$. Because $P$ is composed of two different terms, independent scattering hypothesis is not valid and the probability of a succesive reaction depends on the preceding process. Two body decays, various radiative decays, weak decays, and radiative transitions are studied.

In a system described by $H=H_{0}+H_{1}$, a transition amplitude and probability in the first order of $H_{1}$ from an eigenstate of $H_{0},|\alpha\rangle$ of energy $E_{\alpha}$, to another $|\beta\rangle$ of energy $E_{\beta}$, is computed as, $F_{\alpha, \beta}=\left\langle\beta\left|H_{1}(0)\right| \alpha\right\rangle ; P=\left|F_{\alpha, \beta}\right|^{2} D\left(E_{\beta}-E_{\alpha} ; T\right)$, where $D\left(E_{\beta}-E_{\alpha} ; T\right)=4 \sin ^{2}\left(E_{\beta}-\right.$ $\left.E_{\alpha}\right) T /\left(E_{\beta}-E_{\alpha}\right)^{2} \approx 2 \pi T \delta\left(E_{\beta}-E_{\alpha}\right)$. This leads $P / T=2 \pi \int d \beta \delta\left(E_{\alpha}-E_{\beta}\right)\left|F_{\alpha, \beta}\right|^{2}$ [目]. 1/T correction from the same expression diverges [四]. For the events that the final states interact with or are detected by other microscopic objects, boundary conditions implemented make the correction converge.

The scattering matrix $S[T]$ of satisfying the boundary conditions at $T$ are expressed with Møller operators and satisfy $\left[S(T), H_{0}\right] \neq 0[\mathbb{W}$, []]. A matrix element is written as,

$$
\langle\beta|S(T)| \alpha\rangle=\delta_{\varepsilon}\left(E_{\alpha}-E_{\beta}\right) f(T)_{\alpha, \beta}+\delta f,
$$

$|\alpha\rangle$ and $|\beta\rangle$ are constructed with $\phi_{\text {in }}(x)$ and $\phi_{\text {out }}(x)$, which satisfy,

$$
\lim _{t \rightarrow \mp T / 2}\left\langle\alpha\left|\phi^{f}(t)\right| \beta\right\rangle=\left\langle\alpha\left|\phi_{i n, \text { out }}^{f}\right| \beta\right\rangle,
$$

$\phi^{f}(t)=i \int d^{3} x f^{*}(\vec{x}, t) \partial_{0} \phi(\vec{x}, t)$ with normalized solution $f(\vec{x}, t)$ of a free wave equation [四, 可]. The first term in Eq.([.2) leads $\Gamma T$ and the second term does $P^{(d)}$, which is important for $T \Gamma \approx 0 . \delta f$ and $P^{(d)}$ are governed by the states $\beta$ of $E_{\beta} \neq E_{\alpha}$, which are sensitive to boundary conditions.

\section{Decays}

The interaction Lagrangian of the form,

$$
L_{\text {int }}=\partial_{\mu} G^{\mu}, S_{\text {int }}=\int_{\text {surface }} d S_{\mu} G^{\mu}
$$


where $G^{\mu}$ is a polynomial of fields, does not give bulk effects. $G^{\mu}$ contributes to the surface term, and affects neither the equation of motion nor $\Gamma T$. Neverthless, $G^{\mu}$ modifies the wave function at finite T, and gives $P^{(d)}$. Thus $P^{(d)}$ depends on the wave functions on the surface, which is governed by the states $\beta$ of $E_{\beta} \neq E_{\alpha}$ dtermined by Eq.(ㄸ.3] [四]. Various examples where $G^{\mu}$ is important and $P^{(d)}$ leads observable effects are presented.

$2.1 \pi \rightarrow e+v_{e}$

The pion decay to an electron and neutrino is described by a pion, electron, and neutrino fields, $\varphi_{\pi}(x), e(x)$, and $v(x)$ with an effective action of the form,

$$
S_{i n t}=g \int d x\left[\partial_{\mu}\left(\varphi_{\pi}(x) \bar{e}(x) \gamma^{\mu}\left(1-\gamma_{5}\right) v(x)\right)-i m_{e} \varphi_{\pi}(x) e(x)\left(1-\gamma_{5}\right) v(x)\right],
$$

where the first term is written as the surface integral and does not give bulk effects, and the second term is proportional to the electron mass. The neutrino masses are ignored. $\Gamma$ is from the second term, and is suppressed, known as helicity suppression. $P^{(d)}$ is from the first term and different. Holding lepton universality and being un-suppressed in the electron mode, $P^{(d)}$ is important. In $L \leq L_{0}, P^{(d)}$ should be included, when the theory is compared with experiments. The calculation was made in [[]] using $S[T]$.

The probability of the events that the neutrino is detected at $T$ is given as,

$$
P=N_{3} \int \frac{d^{3} p_{v}}{(2 \pi)^{3}} \frac{1}{E_{v}}\left[\tilde{g}\left(T, \omega_{v}\right)+G_{0}\right]
$$

where $N_{3}=8 T g^{2} \sigma_{v}$, and $L=c T$ is the length of the decay region. $G_{0}$ gives $\Gamma T$, and $\tilde{g}\left(T, \omega_{v}\right)$ gives $P^{(d)}$ which is proportional to the size $\sigma_{v}$ of objects that the neutrino interacts with. $\tilde{g}\left(T, \omega_{v}\right)$ is given in [四] and behaves at large $\omega_{v} T$ as $\frac{2}{\omega_{v} T}, \omega_{v}=\frac{2 E_{v}}{m_{v}^{2}}$. In the region $L \leq 200 M, P^{(d)}$ is larger than the asymptotic values obtained from $\Gamma T$, which is modified by neutrino flavour oscillations [ $[$ ] $]$. Neverthless the value at medium $L$ from the oscillation is much smaller than the value of LSND [ $[\mathrm{d}]$, as is given in Fig. Moreover, the pattern is determined by the neutrino mass and the pion's life time, hence the absolute neutrino mass can be determined.

\subsection{Axial vector meson decays $1^{+} \rightarrow \gamma \gamma$}

Two photon decay rate of axial vector meson vanishes. An effective local interaction of the $1^{+}$ meson $\phi_{1}^{\mu}$ with two photons is expressed with the action,

$$
\begin{aligned}
& S_{\text {int }}=g \int d^{4} x \partial_{\mu}\left(\phi_{1}^{\mu}(x) \tilde{F}_{\alpha \beta}(x) F^{\alpha \beta}(x)\right), \\
& \partial_{\mu} \phi_{1}^{\mu}(x)=0, \tilde{F}_{\alpha \beta}(x)=\varepsilon_{\alpha \beta \gamma v} F^{\gamma v}(x),
\end{aligned}
$$

where $A_{\mu}(x)$ and $F_{\alpha \beta}$ are photon and electromagnetic field strength, and $g$ is the coupling strength. $\Gamma=0\left[\right.$ [Q] but $P^{(d)} \neq 0[$ [四].

$1^{+}$states are formed in $e^{+} e^{-}$or $Q \bar{Q}$ bound states of $L=1$, P-wave. Because P-waves are excited states, it is difficult to study experimentaly for $e^{+} e^{-}$. But those of $C \bar{C}$ have been analyzed. A rate to two gluon decay of color 1 is equivalent to two photon decay, and is estimated from the rate to light hadrons. World averages are

$$
\Gamma_{0}=1.8 \mathrm{MeV}, \Gamma_{1}=0.086 \mathrm{MeV}(0.139 \mathrm{MeV}(B E S S 2)), \Gamma_{2}=0.278 \mathrm{MeV} .
$$


Color 8 contributions and higher QCD corrections leads the rate for $1^{+}$

$$
\Gamma^{(8)}=0.056 \mathrm{MeV}
$$

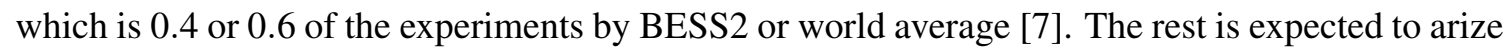
from $P^{(d)}$.

\section{Radiative transitions}

Radiative decays,

$$
\begin{aligned}
& O \rightarrow O^{\prime}+\gamma \\
& O=e^{+} e^{-}, \bar{Q} Q, N, \mu, v
\end{aligned}
$$

are expressed by $E_{1}\left(M_{1}\right)$ or higher multipole transitions, which are expressed by the bulk and surface terms of the effective interactions. $\Gamma$ are determined by the bulk terms and are known well. Giving no-contribution to $\Gamma$, the surface terms have been ignored. Now that gives $P^{(d)}$, and is important. Because $P^{(d)}$ is from the kinetic-enery non-conserving states, the events from $P^{(d)}$ might be hard to identify. Neverthless, they have origins in Schrödinger equation, and play important roles in these processes.

Compton scattering and other radiative transitions

$$
l+\gamma \rightarrow l+\gamma, l+\bar{l} \rightarrow \gamma \gamma, v+\gamma \rightarrow v+\gamma, v+\bar{v} \rightarrow \gamma+\gamma
$$

and others related with QED have been well established [ए0]. Perfect agreements between the theory and experiments there have been obtained as far as $\Gamma T$ are concerned. However, the spectrum from $P^{(d)}$ is quite different from those derived from Fermi's golden rule, $\Gamma T$, and an analysis on total probability at finite $\mathrm{T}$ is made recently [目].

In the measurements of the final states that conserve the kinetic-energy, the events exclude those of $P^{(d)}$ and count only those of $\Gamma T$. These number must be compared with $\Gamma T$. If the number of events are measured without the requirement of kinetic-energty conservation, they are compared with $P=\Gamma T+P^{(d)}$. It is noted, however, that the physical processes in nature are determined by Schrödinger equation and the probability $P=\Gamma T+P^{(d)}$, without excluding $P^{(d)}$. Thus the total probability $\Gamma T+P^{(d)}$ must be used to understand the phycisal phenomena in nature. Enhancement of the events due to $P^{(d)}$ found in various processes will be realized in other processes as well and would give new insights and applications. Experimental identifications of $P^{(d)}$ are highly desired.

\section{Summary and implications.}

$P^{(d)}$ is negligible in atomic transitions [四], but is non-negligible in several cases and has the following unique features.

(1) $P^{(d)}$ has sizable magnitude, inside the region determined by $L_{0}=\frac{\hbar E}{m c^{3}}$. If $L_{0} \geq a$, where $a$ is the spacing of atoms in matter, the process caused by $P^{(d)}$ is enhanced in matter. The kinetic-energy for the event caused by $P^{(d)}$ is not conserved, hence the spectrum includes $h v \neq E_{f}-E_{i}$. 

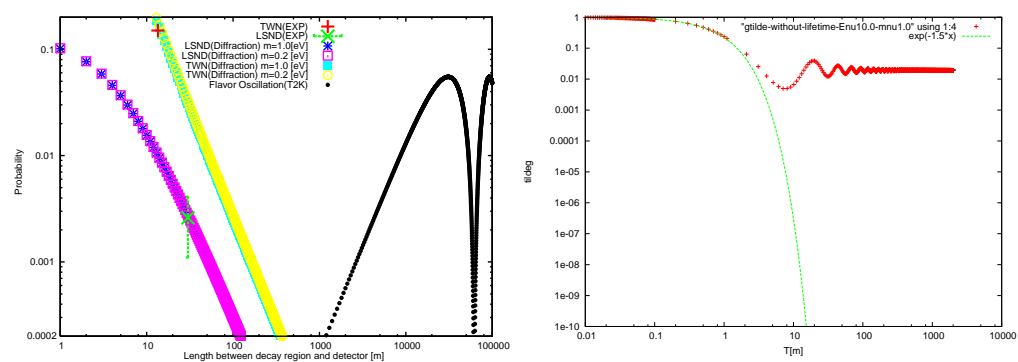

Right: $e^{-T \tilde{g}\left(\omega_{V} T\right)}$. The number of parent from $P^{(d)}$ decreases with $T$ as $e^{-T \tilde{g}\left(\omega_{V} T\right)}$

Figure 1: Left:Relative fractions of the electron mode are computed [3] and compared with experiments [8]. The parameters $m_{v}=0.2 \mathrm{eV} / \mathrm{c}^{2}, E_{v}=250 \mathrm{MeV}$, and $P_{\pi}=2 \mathrm{GeV} / \mathrm{c}(\mathrm{TWN})$, and $m_{v}=0.2 \mathrm{eV} / \mathrm{c}^{2}, E_{v}=$ $60 \mathrm{MeV}$, and $P_{\pi}=300 \mathrm{MeV} / \mathrm{c}$ (LSND). Flavor oscillation from (T2K) parameters $\sin ^{2} \theta_{13}=0.11, \delta m_{23}^{2}=$ $2.4 \times 10^{-3} \mathrm{eV}^{2} / \mathrm{c}^{4}$, and $E_{v}=60 \mathrm{MeV}$ becomes extremely small in $L \leq 1000 \mathrm{M}$ and is insufficient.

Right: $e^{-T \tilde{g}\left(\omega_{v} T\right)}$. The number of parent from $P^{(d)}$ decreases with $T$ as $e^{-T \tilde{g}\left(\omega_{v} T\right)}$

(2) $P^{(d)}$ was derived from Schrödinger equation, and its pattern or distribution is determind by the fundamental parameteres in Lagrangian.

(3) $P^{(d)}$ is extended in large spatial region and gives macroscopic quantum effects. Hence, it afffects physical phenomena of wide area, especially of non-stationary origins. $P^{(d)}$ is inevitable for understanding these phenomena even in macroscopic region.

Acknowledgements. The authors thank Drs. Kobayashi, Maruyama, Nakaya, and Nishikawa for useful discussions on the near detector of T2K experiment, Drs. Asai, Kobayashi, Minowa, Mori, Yamada for useful discussions on interferences.

\section{References}

[1] K. Ishikawa and Y. Tobita. Prog. Theor. Exp. Phys. 073B02, doi:10.1093/ptep/ptt049 (2013).

[2] P.M. Dirac, Pro.Royal Soc. London. A114,243 (1927)

[3] K. Ishikawa and Y. Tobita, "Matter-enhanced transition probabilities in quantum field theory," Annals of Physics (2014) http://dx.doi.org/10.1016/j.aop.2014.02.007 in press.

[4] K. Ishikawa and T.Shimomura, Prog. Theor. Phys. 114, (2005), 1201-1234.

[5] K. Ishikawa and Y. Tobita, Prog. Theor. Phys. 122, (2009), 1111-1136.

[6] K. Ishikawa, T. Tajima, and Y. Tobita, in preparation.

[7] K. Nakamura et al. [Particle Data Group], J. Phys. G 37, 075021 (2010).

[8] C. Athanassopoulos, et al. Phys. Rev. Lett. 75, 2650 (1995), 77, 3082 (1996), 81, 1774 (1998);

G. Danby, et al. Phys. Rev. Lett. 9, 36 (1962).

[9] L. Landau. Sov.Phys.Doclady 60,207(1948); C. N. Yang. Phys. Rev. 77,242(1950).

[10] M. Gell-Mann, phys. Rev. Letters, 6,6,70(1961); L. Rosenberg, Phys. Rev. 129,2786(1963) 